\title{
The enhancement of optical processes near rough surface of metals
}

\author{
G.I. Dovbeshko' ${ }^{1}$ O.M. Fesenko ${ }^{1}$, Yu.M. Shirshov' ${ }^{2}$, V.I. Chegel $^{2}$ \\ ${ }^{1}$ Institute of Physics, NAS of Ukraine, 46, prospect Nauky, 03028 Kyiv, Ukraine \\ E-mail: galyna@ukma.kiev.ua. \\ 2 V. Lashkaryov Institute of Semiconductor Physics, NAS of Ukraine, 41, prospect Nauky, 03028 Kyiv, Ukraine
}

\begin{abstract}
Last decades the enhancement of optical transitions near metal surface was observed under study the optical processes (luminescence, Raman scattering, IR absorption). The effect consists in an essential increase of the intensity of transition (for example, an effective cross-section increases by factor $10^{5} \ldots 10^{11}$ for Raman scattering and $10 \ldots 10^{4}$ for IR absorption) or efficiency of the processes near metal surface (e.g. generation of second harmonic). We have analyzed a various experimental techniques that made it possible to achieve an enhancement in surface enhanced infrared absorption (SEIRA), surface enhanced Raman scattering (SERS), metal-enhanced fluorescence. Under the conditions of our experiment and according to literature data, there was observed enhancement factor which equal to $3 \ldots 20$ for vibrations of various molecular groups in SEIRA. Peculiarity of another optical amplifier, namely, colloidal gold nanoparticles, which were effectively used for enhancement of signal in IR absorption and Raman scattering, metal-enhanced fluorescence, has been studied also. Different roughness of gold surface leads to changes in an enhancement factor. The structural features of bovine serum albumine (BSA) - colloidal gold system and enhancement of guanine that obtained in SEIRA experiment are discussed. Atom force microscopy (AFM) technique was applied to test the roughness of the metal surface. We made an attempt to model the factor of enhancement of electrical field and its frequency dependence for different metal surfaces, and ascertained that silver, gold and copper are the best.
\end{abstract}

Keywords: bovine serum albumin (BSA), Fourier transformed infrared spectroscopy (FTIR), surface enhanced infrared absorption (SEIRA), gold substrate, gold-colloidal nanoparticles, atomic force microscopy (AFM), surface-enhanced Raman scattering (SERS).

Manuscript received 08.10.04; accepted for publication 16.12.04.

\section{Introduction}

The work with biological objects, such as nucleic acids, proteins, lipids, imposes some restrictions on using traditional techniques and makes it necessary to use specific approaches and methods. Even with using the modern equipment, one does not always succeed in obtaining good results. One of the points here is the restricted amount of the available substances to study that the searchers should work with. For this reason, to register the IR spectra and analysis the samples of extremely small amounts of substance, we proposed a modified technique based on enhancement effect of infrared absorption by rough metallic surface (SEIRA). The enhancement of optical process by a factor $10^{2} \ldots 10^{12}$ near rough surface of a metal $(\mathrm{Au}, \mathrm{Ag}, \mathrm{Fe}$, etc.) is already known for twenty years, for both optical transitions in adsorbed molecules and the processes which do not depend on the presence of molecules on the metal surface. For example, these processes are SEIRA, surface-enhanced Raman scattering (SERS), metal-enhanced fluorescence and second harmonics generation that will be discussed below. The effect consists in an essential increase of the intensity of transition (for example, the effective cross-section increases by factor $10^{5} \ldots 10^{12}$ for Raman scattering (RS) and 10 to $10^{4}$ for IR absorption) or efficiency of the process near metal surface. The explanation of the effect is not simple and includes several mechanisms, namely: i) the increase of the electromagnetic field near a rough metal surface or island metal films, ii) the increase of the dipole transition moment of adsorbed molecules, etc. According to [1], the coefficient of the electric field 

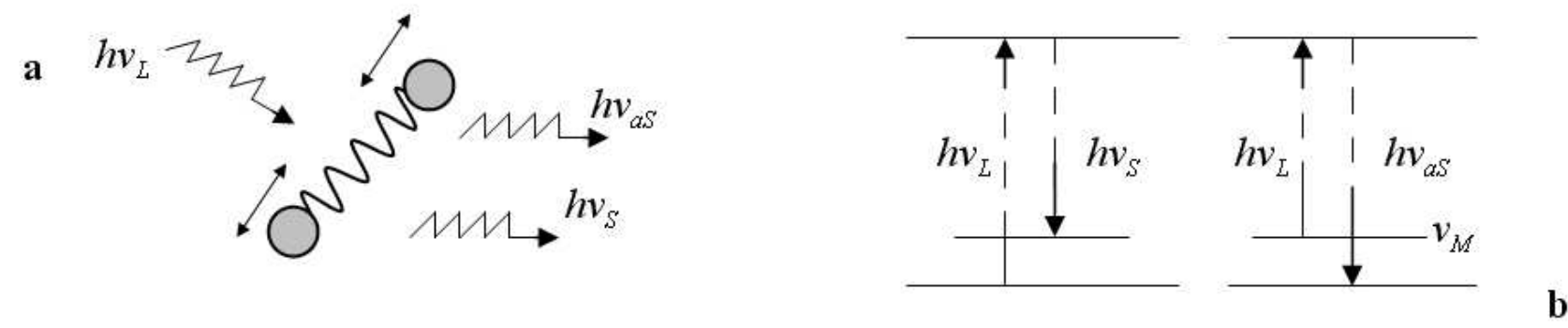

$$
\begin{aligned}
& v_{a S}=v_{L}+v_{M} \\
& v_{S}=v_{L}-v_{M}
\end{aligned}
$$

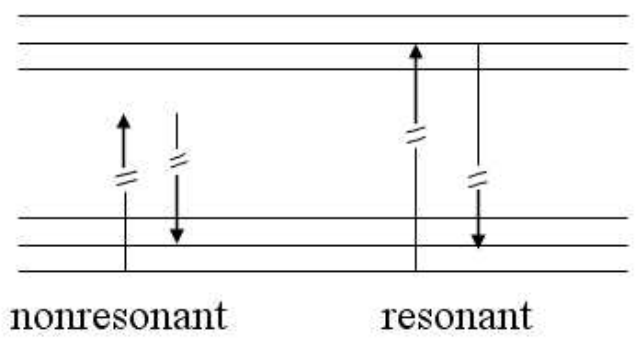

Fig. 1. Schematic diagrams of RS.
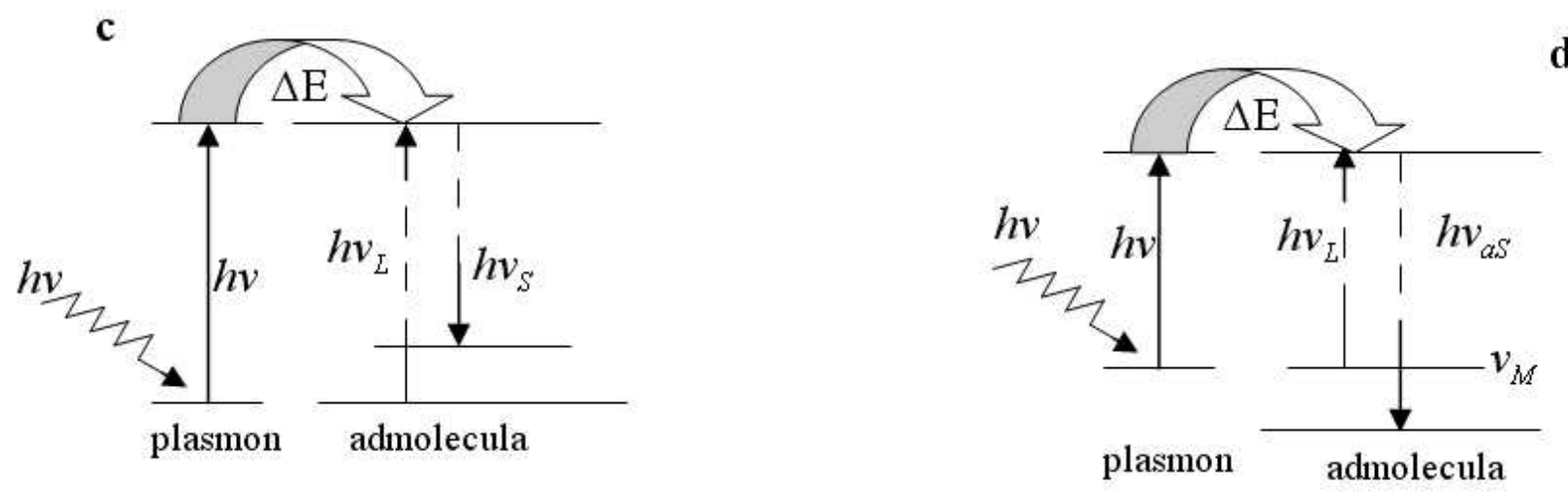

Fig. 2. Schematic diagrams of SERS: a - Stokes scattering; $b$ - anti-Stokes scattering.

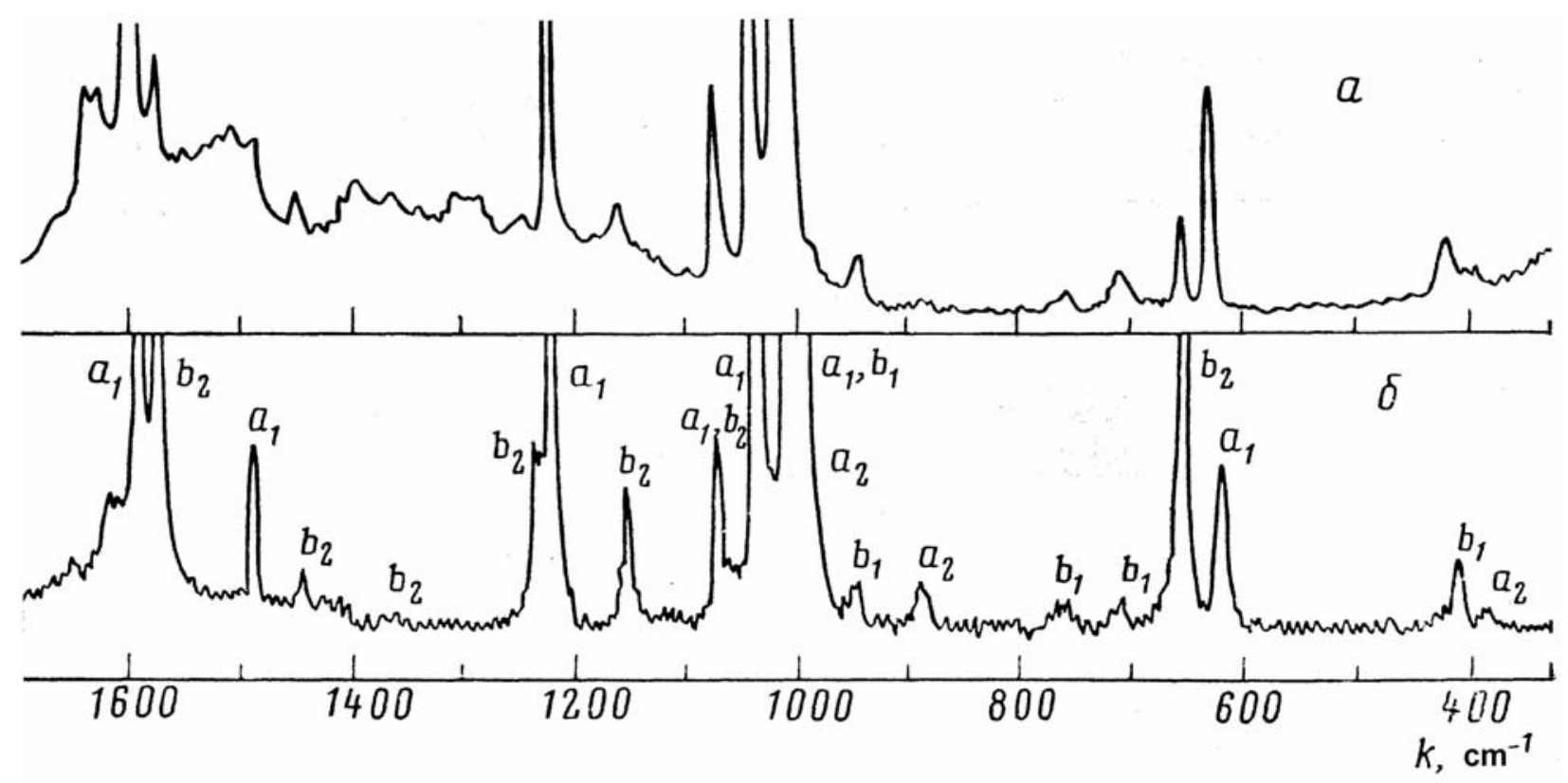

Fig. 3. SERS spectra of pyridine molecules [5] adsorbed on the silver electrode from aqueous solution of $0.1 \mathrm{M} \mathrm{KCl}$ (a) and in $2 \mathrm{M} \mathrm{KCl}(\mathrm{b})$.

(C) 2004, V. Lashkaryov Institute of Semiconductor Physics, National Academy of Sciences of Ukraine 
enhancement is a function of the dielectric permeability of the metal. One should note, however, that the influence of the metal under some conditions and characteristics of the surface or adsorbed substances can lead not only to enhancement but also to weakening the optical transitions of the molecules near the metal surface [2].

\section{SERS mechanisms and enhancement factors}

Before discussing SERS effect, we recall some typical features of Raman effect. In the "photon picture", we considered the Raman effect as a scattering process between a photon and a molecule (see Fig. 1a). Incident photons $h v_{L}$ are inelastically scattered by the molecule and shifted in frequency by the energy of its characteristic molecular vibrations $h v_{M}$. Frequency-shifted scattered photons can occur at lower and higher energies relatively to the incoming photons, depending on whether they interacted with a molecule in the vibrational ground state or an excited vibration state. In the first case, photons lose energy by exciting a vibration and the scattered light appears at a lower frequency $v_{s}$, called the Stokes scattering. By interacting with a molecule in an excited vibrational state, the photons gain energy from the molecular vibrations and the scattered signal appears at higher frequency $v_{a S}$, called the anti-Stokes scattering.

In a "classical view" of the Raman effect, light is an electromagnetic wave that induces the dipole moment in a molecule. The generation of this dipole moment by the incident electric field is modulated by the molecular vibrations, since the polarizibility $\alpha$ is a function of the vibrational coordinate $Q$. Therefore, the induced dipole oscillates not only at the frequency of the incident field, but also at "side bands" at the sum and difference of frequencies between the incident light and the molecular vibrations. This results in the appearance of the Stokes and anti-Stokes "side bands" in the reradiated light. Fig. 1b shows the scheme of RS in a molecular energy level diagram together with typical Raman spectra. The Raman effect allow us to probe vibrational levels of the molecule, which depend on the type of atom and their bond strengths as well as arrangements in a specific molecule. Therefore, a Raman spectrum provides a structural "fingerprint" of a molecule.

SERS is a spectroscopic technique that combines modern laser spectroscopy with the exciting optical properties of metallic nanostructures, resulting in strongly increased Raman signals when molecules are attached to nanometer-sized gold and silver structures. The effect provides the structural information content of Raman spectroscopy together with ultrasensitive detection limits, allowing Raman spectroscopy of single molecules. Since SERS takes place in the local fields of metallic nanostructures, the lateral resolution of the technique is determined by the confinement of the local fields, which can be two orders of magnitude shorter than the diffraction limit. Moreover, SERS is the analytical technique, that can give information about surface and interface processes.

In SERS, molecules are attached to a metallic "nanostructure". The effect was discovered when studying pyridine $\left(\mathrm{C}_{5} \mathrm{H}_{5} \mathrm{~N}\right)$ and cyanide $\left(\mathrm{CN}^{-}\right)$ adsorbed on electrochemically roughened silver electrodes [3, 4]. In 1979, enhanced RS signals from pyridine were also measured in silver and gold aqueous colloidal solutions. These experiments showed that SERS is a "nanostructure effect" rather than a "surface effect" and provided the first clear experimental demonstration of the important role of surface plasmon resonances in SERS (Fig. 2).

Until now, the SERS effect was observed for more than 100 molecules of various structures and properties. Among them, there are molecules with double and triple bonds, atom and molecular ions, and a number of atomic molecules, polymers, and biopolymers. Some of the molecules [e.g., pyridine (Fig. 3) [5] and $\mathrm{CN}^{-}$] are chemically adsorbed, other (e.g., CO) are physically adsorbed.

The shape of RS spectra of admolecules represents the following factors: 1) type of molecules; 2) type of metal (adsorbent); 3) peculiarities of dielectric medium in which molecules are absorbed; 4) structure of non-homogeneous medium (roughness of the surface).

Different theories for physical mechanisms of SERS effect are listed in literature [6].

1.SERS is considered as resonance RS that is registered upon the shift and widening of electronic energy levels of adsorbed molecules. One of the mechanisms of the effects is an interaction of electronic surface mode with surface plasmons. Other possible mechanism is a shift of energy levels that results in formation of a metal-molecule complex with chemical bonds.

2. Theory of modulated surface reflection is based on the assumption that there is vibration of the charge of a chemical molecular bond at the metal surface, which modulates the reflection coefficient of the metal surface due to modulation of the metalmolecule complex permeability.

3. Theory of dipole imaging field uses an idea of increasing the polarizability of molecules attached to the metal surface due to the influence of the dipole imaging field.

4. Microscopic theories of SERS effects that are based on the description of the process of excitation and relaxation of electronic and vibronic states in metal and molecules.

5. Electromagnetic SERS theory based on electronic vibration in restricted submicroscopic structures (roughness of the surface or colloidal particles). As the fact, a theory of the last class based on phenomena of increase of a "local" field, which influences the adsorbed molecule and appears in the 


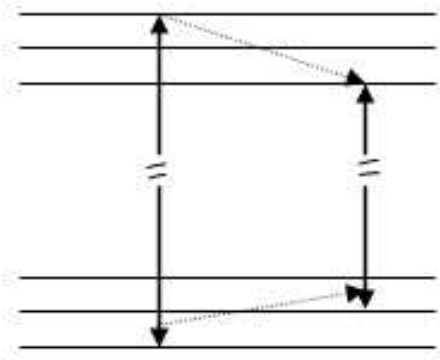

Fig. 4. Schematic diagram of fluorescence.

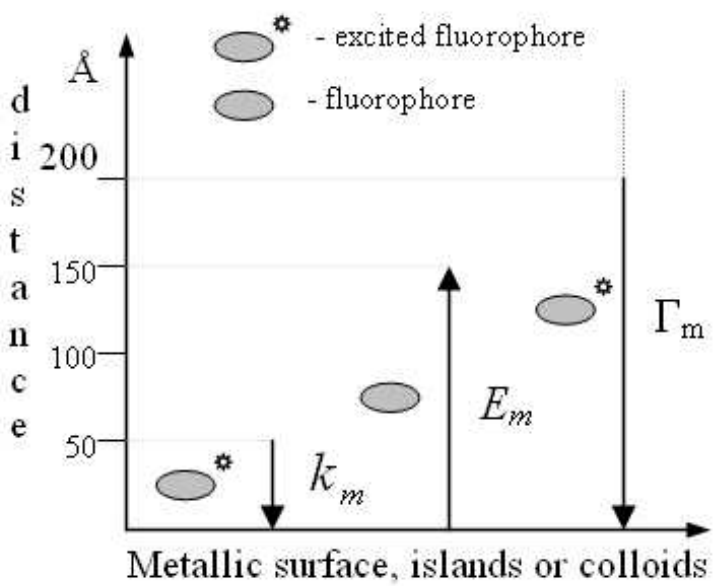

Fig. 5. Predicted distance dependences for a metal surface on the transition of fluorophore. The metallic surface can cause Forsterlike quenching with a rate, $k_{m}$, can concentrate the incident field, $E_{m}$, and can increase the radiative decay rate, $\Gamma_{m}$.

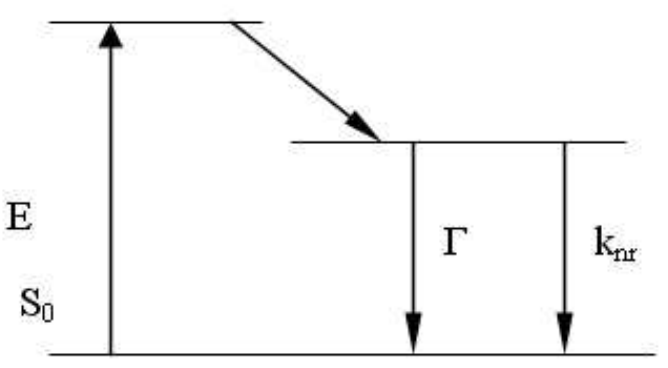

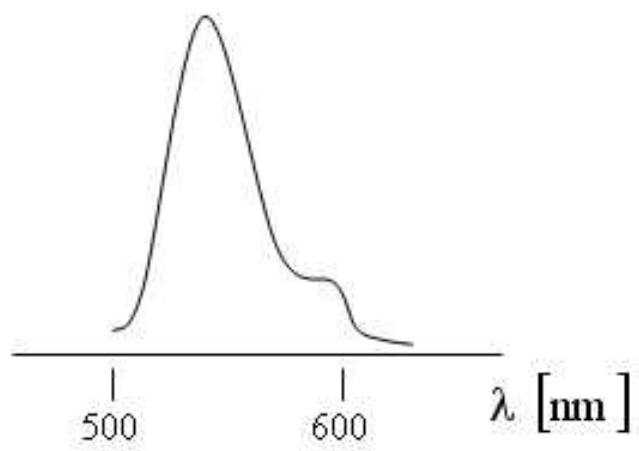

With metal

No metal

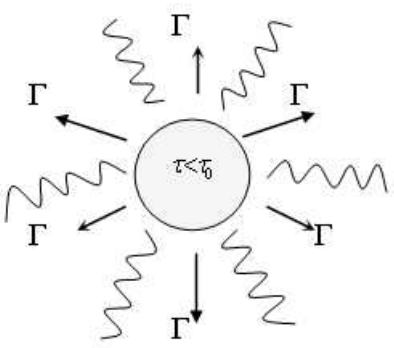

High photonic

mode density

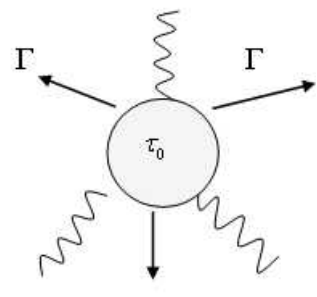

$\Gamma$

Low photonic mode desity

Fig. 6. High and low photonic mode densities in the presence and absence of metal, respectively.

Metallic particles, Islands or colloids

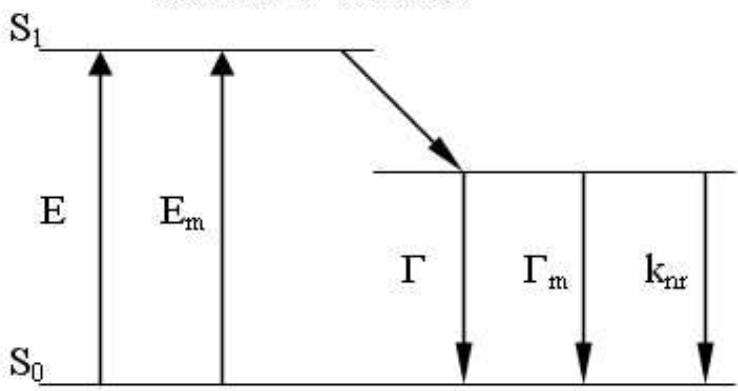

Fig. 7. Classical Jablonski diagram for the free space condition and the modified form in the presence of metallic particles, islands or colloids. $E$ - excitation, $E_{m}$ - metal enhanced excitation rate, and $\Gamma_{m}$-radiative rate in the presence of metal. 
system of submicroscopic particles of a noble metal. The theory of the last class describes all the SERS experiments in details. For example, the mechanisms, which are the bases of the theories (1)-(4), are not connected with the level of surface roughness. Therefore these theories point to the possibility for the SERS effect to be realized on a smooth surface. This conclusion contradicts to the experiment. Cross-section of the Raman process equaled to the density of the scattered power per unit of space angle could be presented by the following formula:

$\left(\frac{d \sigma}{d \Phi}\right)_{R S}=k_{S}^{4}\left|\vec{d}_{S}\right|^{2}$,

where $k_{S}=\omega_{S} / c, \quad \omega_{S}=\omega_{i}-\Omega, \quad \vec{d}_{S}=\vec{r}^{\prime} \vec{E}_{i} \quad$ is the amplitude of the Stokes component of molecular dipole, $\hat{r}^{\prime}-\mathrm{RS}$ tensor, $E_{i}, \omega_{i}$ - amplitude and frequency of exciting light wave. In the case of RS by the molecule adsorbed on the rough metal surface, the amplitude of the Stokes dipole is equal to $\vec{d}_{S}=\widehat{r}^{\prime} \vec{E}_{l o c}$, where $\widehat{r}^{\prime}$ is the RS tensor of the adsorbed molecule (where the possible shift of molecular levels is taken into account), local field $\vec{E}_{l o c}=L\left(\omega_{i}\right) \vec{E}_{i}$. The effective dipole moment that radiates with $\omega_{S}$ frequency is equal to:

$\vec{d}_{e f f}\left(\omega_{s}\right)=L\left(\omega_{S}\right) \vec{d}_{S}=L\left(\omega_{S}\right) L\left(\omega_{i}\right) \vec{r}^{\prime} \vec{E}_{i}$,

where $L\left(\omega_{S}\right)$ includes the contribution of dipole moments of geometrically bounded metal structures.

Taking into account the surface geometry only one component of $\vec{E}_{i}$ excites plasmons, then it is possible to simplity the expression for $\vec{d}_{s}$ in direction where $d_{s}, r$ are the components of corresponding vector values. Substituting (2) into (1), we get the cross-section of RS molecule fixed at the rough metal surface (or on the surface of colloidal particles):

$\left(\frac{d \sigma}{d \Phi}\right)_{S E R S}=\left(\frac{d \sigma}{d \Phi}\right)_{R S}\left|\frac{\vec{r}^{\prime}}{\widehat{r}}\right|^{2}\left|L\left(\omega_{S}\right)\right|^{2}\left|L\left(\omega_{i}\right)\right|^{2}$.

Neglecting the changes of the RS tensor during adsorption $\left(\widehat{r}^{\prime}=r\right)$, we get the next expression for the enhancement factor of RS that follows from (3):

$$
\left(\frac{d \sigma}{d \Phi}\right)_{S E R S} /\left(\frac{d \sigma}{d \Phi}\right)_{R S}=G=\left|L\left(\omega_{i}\right)\right|^{2}\left|L\left(\omega_{s}\right)\right|^{2}
$$

As appears from [7] the enhancement factor $G$ could be approximately $10^{6} \ldots 10^{8}$ for certain metals under resonance conditions, however, now the experimental enhancement factor could reach to $10^{11}$ [4].

\section{Metal-enhanced fluorescence}

Fluorescence spectroscopy is a widely used research tool in biochemistry and molecular biology. Fluorescence has also become the dominant method enabling the revolution in medical diagnostics, DNA sequencing and genomics. To date, all the fluorescence observables including spectral shifts, anisotropies, quantum yields and lifetimes, have already been used in basic and applied studies of fluorescence.

Fig. 4 shows the scheme of fluorescence in a molecular energy level diagram together with typical fluorescence. In many cases, fluorescence spectra are relatively "broad bands" and do not provide so much structural information on the molecule.

Whereas the demonstrations and applications of metal-enhanced fluorescence (MEF) are mostly new, the theory of fluorescence enhancement with metallic surfaces and particles has developed since the 1980s, in which the effects are caused by at least three known mechanisms [8] (Fig. 5). One is energy transfer quenching, $k_{m}$, to the metal with a $d^{-3}$ dependence [9]. This quenching can be understood by damping the dipole oscillations near metal. The second mechanism is increasing the emission intensity due to caused by metal increasing the local incident field on the fluorophore, $E_{m}$, with a maximum theoretical enhancement effect predicted to be near 140 [10]. This effect was been observed for metal colloids and is appropriately called the lightening rod effect [11, 12]. This enhancement can be understood as caused by the metal particles concentrating the local field and subsequent increasing the rate of excition. The third mechanism is that adjacent metal can increase the intrinsic radiative decay rate of fluorophore, $\Gamma_{m}$, that is, to modify the rate at which a fluorophore emits photons (Fig. 6) [13, 14].

To better understand this concept, it is informative to consider the Jablonski diagram for fluorophores under the free-space condition and the modified form when being in the close proximity to a conducting metallic particle or colloids (Fig. 7).

Absorption of a photon transfers the fluorophore to the first excited singlet state $\left(S_{1}\right)$ and then the excited molecules can emit a photon at a rate $\Gamma$, or return to the ground state emitting a photon at a rate $\Gamma$, or return to the ground state by a non-radiative decay process, with a rate $k_{n r}$. Other deactivation processes are also possible to depopulate the $S_{1}$ level, such as quenching processes with a rate $k_{q}$. The quantum yield, $Q_{0}$, of a fluorophore shows a competition between radiative decay and nonradiative processes:

$$
Q_{0}=\frac{\Gamma}{\Gamma+k_{n r}+k_{q}} \text {. }
$$




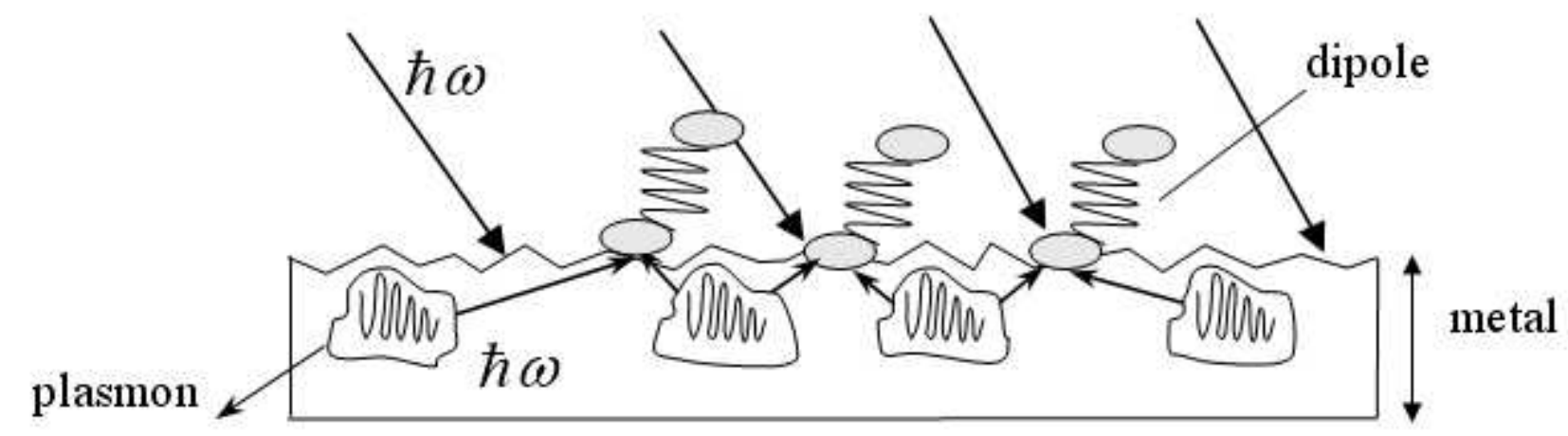

Fig. 8. Scheme of the mechanism for the infrared absorption enhancement at the metal surface (SEIRA).

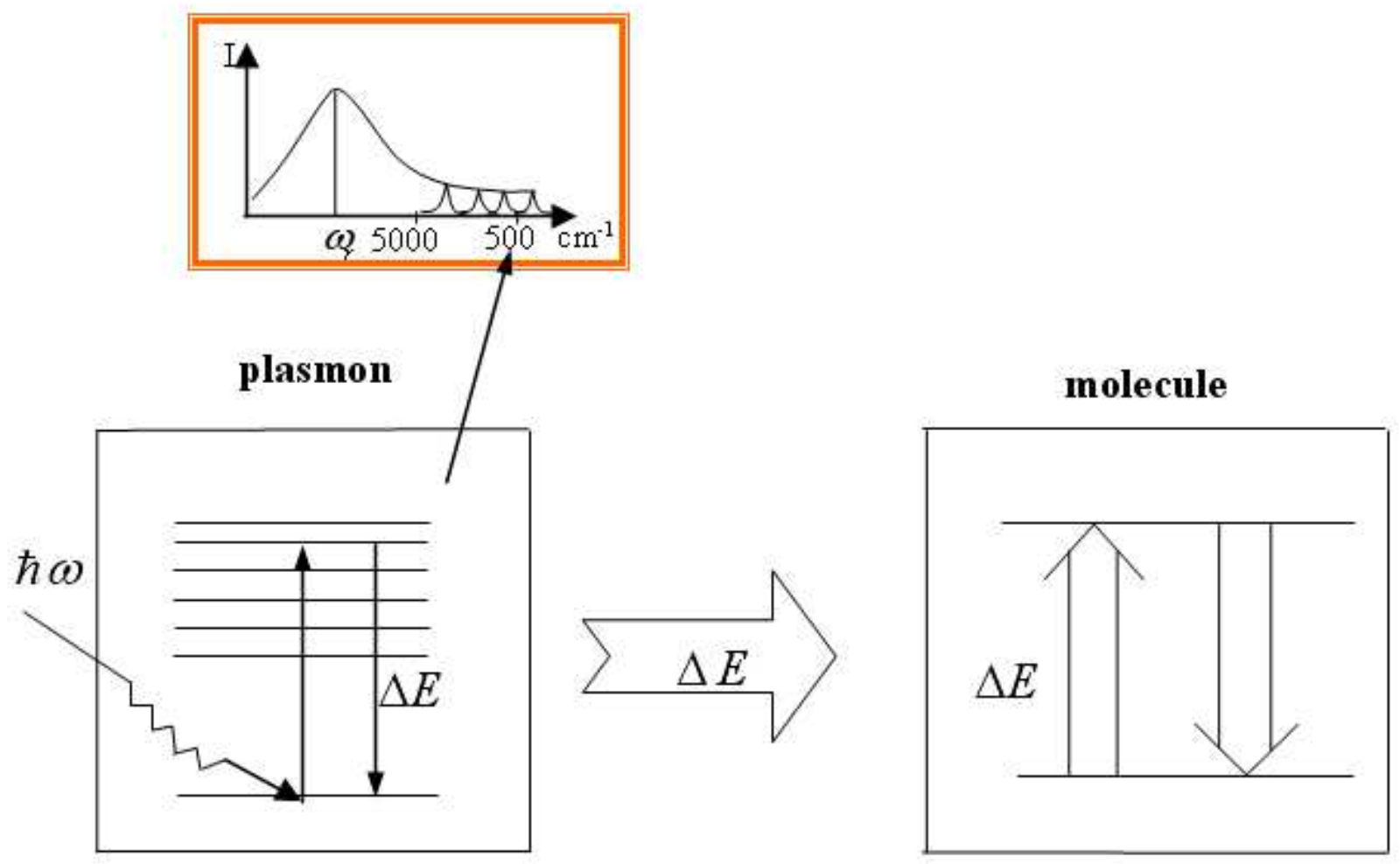

Fig. 9. Energy scheme of the mechanism of the enhancement effect.

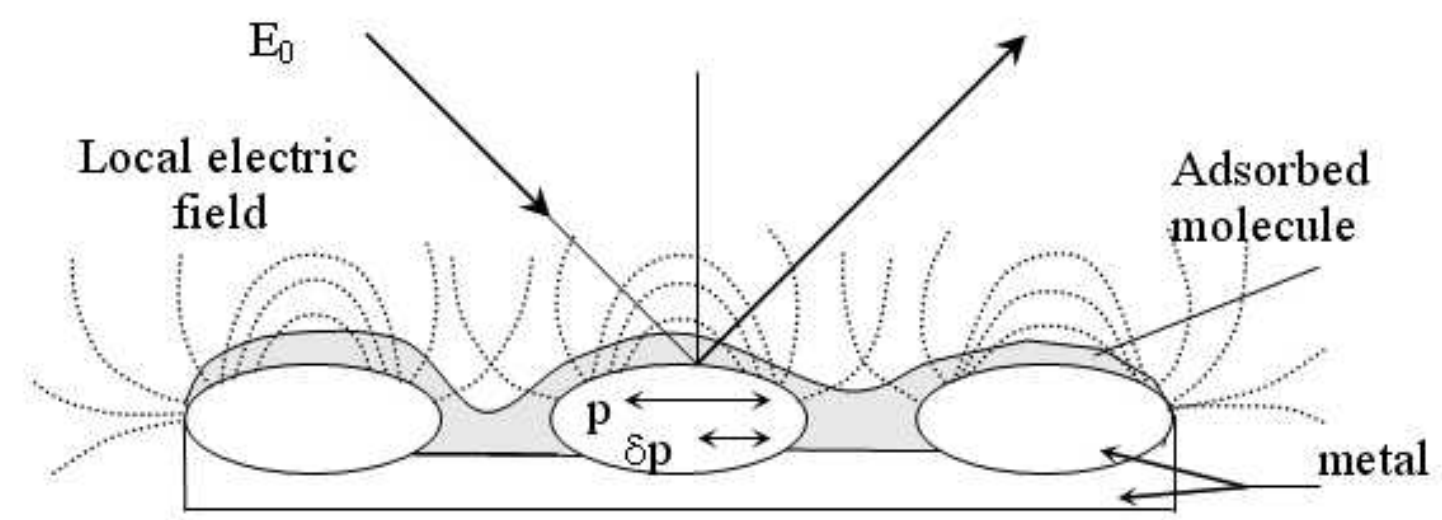

Fig. 10. Scheme of the SEIRA experiment in reflectance geometry.

(C) 2004, V. Lashkaryov Institute of Semiconductor Physics, National Academy of Sciences of Ukraine 
The fluorescence lifetime, or decay time, is the average time for an ensemble of fluorescent molecules to remain in the $S_{1}$ state:

$\tau_{0}=\frac{1}{\Gamma+k_{n r}+k_{q}}$.

The fluorophores immediate environment strongly influences its fluorescence lifetime and intensity, but does not significantly alter $\Gamma$. These changes occur because of changes in $k_{n r}$ or $k_{q}$. If $Q_{0}$ is relatively small, then $k_{n r}+k_{q}$ is much larger than $\Gamma$. If $Q_{0}$ is high, then $\Gamma$ is larger than $k_{n r}+k_{q}$. Hence, we can modify the spectral changes of fluorophores by modifying $k_{n r}$ or $k_{q}$. Invariable $Q_{0}$ and $\tau_{0}$ change together.

Modification of $\Gamma$ is usually not considered because this rate is determined by the transition probability and oscillator strength of the $S_{1} \rightarrow S_{0}$ transition. When fluorophores are placed at suitable distances from metallic particles or surface, fluorophores can undergo modifications of their radiative decay rates, $\Gamma_{m}$, where an increase in $\Gamma_{m}$ results in an increase in fluorescence intensity, $Q_{m}$, and reduction in lifetime $\tau_{m}$, which is converse to the free-space condition in which both change in unison

$$
\begin{aligned}
Q_{m} & =\frac{\Gamma+\Gamma_{m}}{\Gamma+\Gamma_{m}+k_{n r}+k_{q}}, \\
\tau_{m} & =\frac{1}{\Gamma+\Gamma_{m}+k_{n r}+k_{q}} .
\end{aligned}
$$

An ability to modify and control the radiative decay rate $\left(\Gamma+\Gamma_{m}\right)$ can have profound implications for the use of fluorescence in basic research and its applications. The modification and control of the radiative rate has been recently named radiative decay engineering (RDE) by [13], but they have now tended toward using MEF, terminology that additionally accounts for the other socalled lightening rod fluorescence enhancement effect.

At this point, it is informative to consider how modifications of both $E_{m}$ and $\Gamma_{m}$ are likely to influence other well-characterized free-space fluorescence phenomenon.

\section{Theoretical interpretation and mechanisms of SEIRA effect}

In modern biological experiments, it is very important to perform a conformation analysis of biological molecules under external stimuli: $\mathrm{pH}$, interaction with small and big molecules, temperature, etc. The FTIR method, namely SEIRA is a good tool for the experiments on conformational analysis of biological molecules in real time and in situ [15].

The interpretation of the effect of enhancement is presented in [16] in a general form. It includes two possible ways: enhancement of the electromagnetic field due to interaction with local (surface) plasmon oscillations which emerge on the imperfections of the metal surface, and specific increase of the dipole transition moments of the molecules adsorbed on the metal surface (Fig. 8).

The theoretical interpretation of the effect is connected with the enhancement of the external electric field due to excitation of local (surface) plasmon vibrations at essential curvature of rough surface or on metal particles. In such systems, according to [5], the electric field in the point of space $r$ could be written as follows :

$E(r, t)=\exp (-i \omega t)\left[E_{0}(\omega)+E_{1}(r, \omega)\right]$,

where $\omega$ is the frequency, $t$ is the time. The additional term $E_{1}(r, \omega)$ is connected with the excitation of local (surface) plasmon vibrations. The enhancement of the external field can be presented as

$E_{\alpha}(\mathrm{r}, \omega)=g_{\alpha \beta}(r, \omega) E_{0 \beta}(\omega)$,

where $g_{\alpha \beta}(r, \omega)$ is the enhancement coefficient. For matrix elements of the dipole transition moment for the transition between the states $i$ (with energy $\varepsilon_{i}$ ) and $f$ for the adsorbed molecule of nucleic acid, we have

$\left\langle f\left|d_{\alpha}(r)\right| i\right\rangle=h_{\alpha \beta}{ }^{(f i)}(r)\left\langle f\left|d_{0, \beta}\right| i\right\rangle$,

where $\left\langle f\left|d_{0}\right| i\right\rangle$ is the matrix element of the dipole transition moment for an isolated molecule. According to the classical approximation, the matrix elements of the Hamiltonian describing the interaction of the adsorbed molecule with field are:

$\left\langle\langle f \mid H(r, \omega) i\rangle=-\left\langle f\left|d_{\alpha}(r)\right| i\right\rangle E_{\alpha}(r, \omega)=-h_{\alpha \beta}{ }^{(f i)}(r) g_{\alpha \gamma}\right.$

$(r, \omega)\left\langle f\left|d_{0, \beta}\right| i\right\rangle E_{0, \gamma}(\omega)$.

Having performed substitution from Eq. (12) into the expression

$\omega_{f i}(r, \omega)=(2 \pi / \hbar)|\langle f|H(r, \omega)| i\rangle|^{2} \delta\left(h \omega-\varepsilon_{f}+\varepsilon_{i}\right)$,

for the rate of the $i \rightarrow f$ transition, multiplied the result by the energy $h \omega$ of absorbed quantum, and divided by the density of the incident energy flow $c E_{0}{ }^{2} / 8 \pi$ (where $c$ is the light velocity), we obtain the expression for the effective cross-section of light absorbance by the adsorbed molecule:

$\sigma_{\alpha}(r, \omega)=\frac{16 \pi^{2} \omega_{f i}}{c E_{0}^{2}}|\langle f|H(r, \omega)| i\rangle|^{2} \delta\left[h\left(\omega-\omega_{f i}\right)\right] \sim$

$\sigma_{\alpha}{ }^{(0)}|g(r, \omega)|^{2}|h(r)|^{2}$,

where $\sigma_{\alpha}^{(0)}$ is the cross-section for the case when metal is absent $\left(d=d_{0}, E=E_{0}\right)$. The right hand part of Eq. (14) is the estimation of the cross-section by its order of magnitude.

The energy scheme of the mechanism of the enhancement effect is presented in Fig. 9. Under the conditions of IR experiment, we work at the wing of the plasmon resonance for the golden substrate, as it is shown in the insertion of Fig. 9. The energy is transferred from the photon to local plasmon oscillations. The plasmon oscillations have resonant 
character, and their frequency is determined by the form of the boundary. The surface charge that emerges on the roughnesses causes the Coulomb returning force. The energy is transferred from plasmon vibrations to the adsorbed molecules, which causes the increased molecular absorbance.

\section{Experimental methods of the use of SEIRA effect}

In literature, there are presented three experimental realizations of the SEIRA method.

\subsection{SEIRA method in reflectance geometry}

First - the SEIRA method in reflectance geometry (Fig. 10). The enhancement occurs due to increasing of the intensity of electric field upon excitation of local plasmons in the rough metal film. Earlier [17] we studied the validity of using the effect of the infrared absorption enhancement by metal surface for nucleic acids and lipids deposited onto the golden substrate as compared with the experiment in transmittance mode.

The results show that when using the SEIRA method for the study of IR absorption of nucleic acids (and similarly for lipids [18]), no deformation of contour of the absorption bands is observed as compared with the case of the neutral $\mathrm{CaF}_{2}$ substrate. Some frequency shifts are present, but for the most part of the bands they are small $\left(1 \ldots 2 \mathrm{~cm}^{-1}\right)$. This allows us to make conclusion about the possibility to use this method for solving the problems of this type [18]. The experimentally observed coefficients of the integral intensity enhancement for the absorption bands that correspond to vibrations of various molecular groups of DNA were found to be equal to $3 \ldots 5$ as dependent on the thickness of the sputtered gold layer (Table 1) [19].

Surface structure and geometry influence on enhancement. Thus, we have analyzed two surfaces of different geometry to study the surface influence on enhancement value (Fig. 11). Glass plates with the sputtered gold layer with the thickness $350 \AA$ (substrate $a$ ) and $170 \AA$ (substrate $b$ ) were used as substrates. The roughness of the surface $a$ was arranged almost uniformly on the substrate surface and was close to $19 \AA$ Á. According to the literature data, the roughness and heterogeneity of submicroscopic scale are of great concern in appearance of surface enhancement of IR absorption.

Table 1. The enhancement factor for the integral intensity of the DNA absorption bands taken from our experiments on SEIRA [18] and calculation data based on permeability $\left(\mathrm{g}=\varepsilon^{\prime} / \varepsilon^{\prime \prime}\right)$ for solid gold at appropriate frequencies [19].

\begin{tabular}{|c|c|c|c|c|}
\hline $\begin{array}{c}\text { DNA on } \mathrm{CaF}_{2}, \\
\text { band position, } \mathrm{cm}^{-1}\end{array}$ & $\begin{array}{c}\text { DNA on } \mathrm{Au}, \\
\text { band position, } \mathrm{cm}^{-1}\end{array}$ & \multicolumn{2}{|c|}{ Enhancement factor } & Assignment \\
\hline 3350 & 3345 & experiment & calculation & \\
\hline 1655 & 1653 & 5 & 48 & $\begin{array}{c}\text { Str. O-H, N-H, C- } \\
\mathrm{H}\end{array}$ \\
\hline 1238 & 1230 & 3 & 14 & $\begin{array}{c}\mathrm{C}=\mathrm{O}, \mathrm{C}=\mathrm{N}, \mathrm{N}-\mathrm{H} \\
\text { adenine, thymine, } \\
\text { guanine, cytosine }\end{array}$ \\
\hline & & & 9 & str. $\mathrm{PO}_{2}^{-}$asym. \\
\hline
\end{tabular}

Table 2. The enhancement factor for the integral intensity of guanine absorption bands presented the first in comparison with calculation data.

\begin{tabular}{|c|c|c|c|c|c|}
\hline \multirow{2}{*}{$\begin{array}{c}\text { Guanine on } \\
\mathrm{CaF}_{2} \\
\text { band position, } \\
\mathrm{cm}^{-1}\end{array}$} & \multirow{2}{*}{$\begin{array}{c}\text { Guanine on } \\
\text { Au, } \\
\text { band position, } \\
\mathrm{cm}^{-1}\end{array}$} & \multirow{2}{*}{$\begin{array}{c}\text { Guanine, } \\
\text { calculated } \\
\text { band position } \\
{[20], \mathrm{cm}^{-1}}\end{array}$} & \multicolumn{2}{|c|}{ Enhancement factor } & \multirow[b]{2}{*}{ Assignment } \\
\hline & & & $\begin{array}{c}\text { experiment } \\
g^{2}\end{array}$ & $\begin{array}{c}\text { calculation } \\
g^{2}\end{array}$ & \\
\hline 1698 & 1695 & 1689 & 4.9 & 12.3 & $\operatorname{def} \mathrm{NH}$, str CN \\
\hline 1633 & 1633 & 1633 & 5.0 & 11.6 & $\operatorname{def} \mathrm{NH}$, str $\mathrm{CN}$ \\
\hline 1521 & 1519 & 1569 & 4.4 & 9.6 & str CN \\
\hline 1473 & 1473 & 1526 & 5.6 & 8.3 & str $\mathrm{CN}, \operatorname{def} \mathrm{CNH}$ \\
\hline 1462 & 1463 & 1449 & 4.6 & 9.0 & strCN, str CC \\
\hline 1417 & 1417 & 1398 & 6.2 & 8.4 & $\operatorname{def} \mathrm{CNH}, \operatorname{str} \mathrm{CN}$ \\
\hline 1372 & 1372 & 1369 & 6.0 & 7.8 & $\begin{array}{c}\text { str } \mathrm{CN}, \text { str } \mathrm{CC}, \\
\text { def } \mathrm{CNH}\end{array}$ \\
\hline 1342 & 1342 & 1344 & 3.1 & 3.6 & def $\mathrm{CNH}$ \\
\hline 1314 & 1314 & 1315 & 7.3 & 7.3 & $\operatorname{def} \mathrm{CNH}$ \\
\hline 1118 & 1118 & 1153 & 3.1 & 5.3 & def NH, str CN \\
\hline
\end{tabular}


a

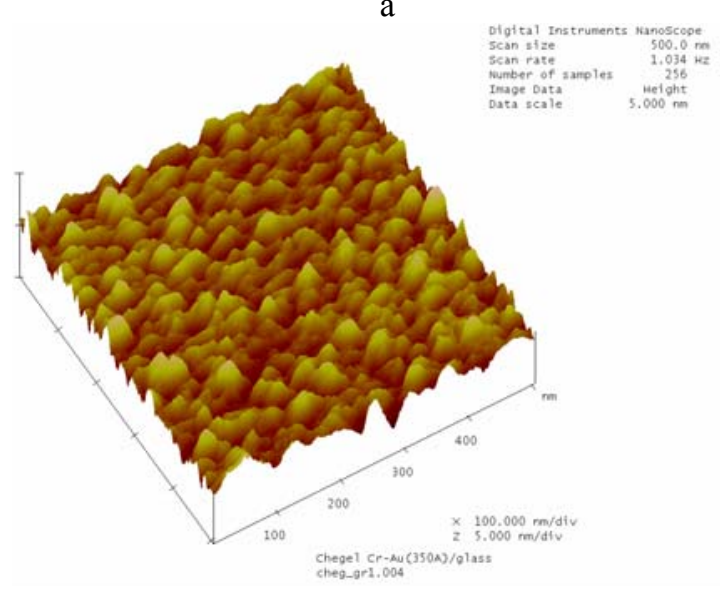

b

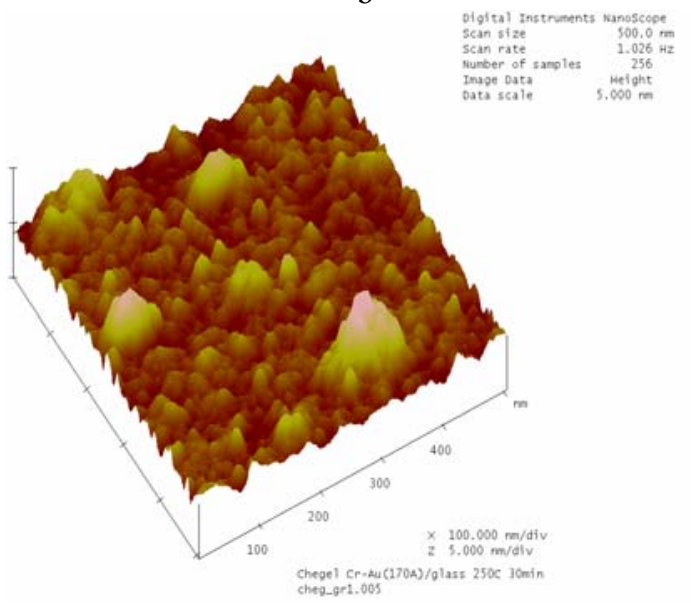

Fig. 11. Image of gold surface in SEIRA experiment (a) and after annealing $250^{\circ} \mathrm{C}$ (b)

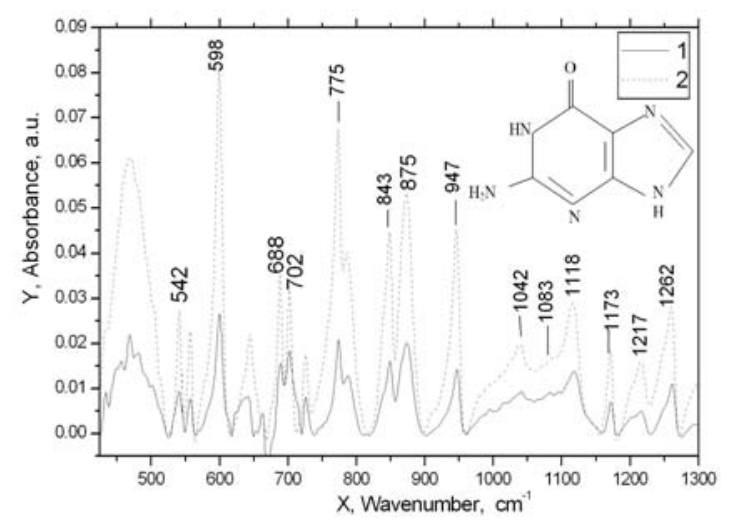

Fig. 12. SEIRA spectra of guanine: 1 - on the gold substrate b, 2 - on the gold substrate a.

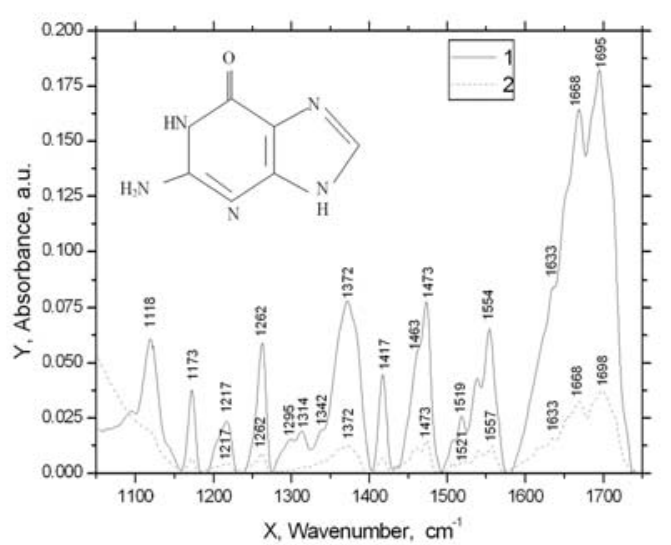

Fig. 13. SEIRA spectra of guanine: 1 - on the gold substrate a, $2-$ on $\mathrm{CaF}_{2}$.

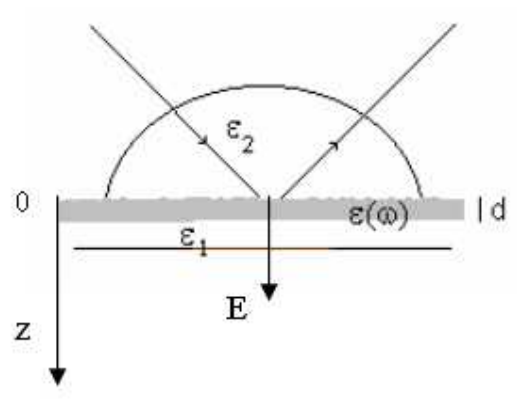

Fig. 14. Scheme of the ATR method in the Kretschmann geometry.

That is why the substrate $b$ was annealed at $2500^{\circ} \mathrm{C}$ to obtain another surface geometry and size of heterogeneities. This resulted in their non-uniform distribution on the surface of substrates and their aggregation into separate groups. The height of some aggregates reached $40 \AA$, though the average roughness of substrates $b$ was close to $10 \AA$. The annealing also caused the decrease of the average radius of the horizontal cross- section of the roughness and of their quantity in comparison with the substrate $a$.

Spectra were collected with IFS-48 Bruker instrument in IR reflectance mode for the guanine on gold substrate. The reflectance attachment used in the experiment has the light incidence angle close to $16.5^{\circ}$ in [17]. The spectra were evaluated using Opus 2.2 software. The position of the bands was estimated 
using standard method. The intensity of the bands was normalized to the band with maximal intensity in each spectrum.

To study the surface geometry influence on the enhancement effect, the samples of aqueous solution of guanine $\left(\mathrm{C}_{5} \mathrm{H}_{5} \mathrm{ON}_{5}\right)$ with the concentration of $0.5 \mathrm{~g} / 1$ were deposited on the substrates $a, b$ and on $\mathrm{CaF}_{2}$ and then dried lyophilically. The samples were deposited in such a way to obtain the layers of the same size and thickness. When comparing the surface geometry influence, it was ascertained that the substrate $a$ causes a greater enhancement than the substrate $b$ does (Fig. 12). This fact could be explained by local plasmon vibrations that appear on the surface. They are resonances determined by surface geometry, and their frequency is defined by the shape of frontier that forms the surface charge. For the surfaces with variable curvature, the resonance enhancement of the electric field is accompanied by an additional field enhancement close to the point of heterogeneity. Indeed, when creating the roughness of surface with scale smaller than the light wavelength, that increases the possibility of formation of local plasmon vibrations, but this is accompanied by rapid decreasing the lifetime of surface electromagnetic waves and enhancement of the electric field due to them, which is determined by the processes of their radiation decay and scattering by the roughness [5].

In Table 2, we present the experimental (Fig. 13) and calculated coefficients of integral intensity enhancement of the absorbance bands of various guanine molecular groups adsorbed onto the gold substrate and $\mathrm{CaF}_{2}$. The experimentally observed enhancement coefficients are determined as the ratios of integral intensities of the absorbance bands inherent to corresponding guanine molecular groups. The calculated enhancement coefficients are obtained as the ratio of real and imaginary parts of the dielectric permeability of the golden substrate at corresponding frequencies. The experimental frequencies of guanine from our experiment and calculated by [20] are also summarized in Table 2 .

\subsection{SEIRA method in the geometry of attenuated total reflectance (ATR)}

The second method realizes the enhancement in the geometry of attenuated total reflection (ATR). There exists the possibility of a resonant enhancement of the electric field near ideally flat metal surface caused by nonradiational surface electromagnetic wave that is excited when using the ATR method (Fig. 14).

One can show that for the prism-metal-dielectric medium configuration, where the media have thedielectric permeabilities $\varepsilon_{2}, \varepsilon(\omega)$, and $\varepsilon_{1}$, respectively, and their boundaries are located at $z=0$ and $z=d[1]$.

If flat light wave with the amplitude $E_{0}(\omega)$ falls from the internal part of the prism onto its surface $z=0$ at the angle $\theta_{i}>\arcsin \left(\varepsilon_{2} / \varepsilon_{1}\right)$, then for $x$-component of the field on the surface of metal $z=d=0$ within the framework of macroscopic electrodynamics for $\omega=\omega_{s}\left(k_{\Uparrow}\right)$ we obtain

$\left|\frac{E_{x}(d)}{E_{0, x}}\right|^{2}=\frac{16}{1+\left(\alpha_{1} / \beta_{2}\right)^{2}} \mathrm{e}^{-2 h_{1} d}\left|\frac{\varepsilon^{\prime}}{\varepsilon^{\prime \prime}}\right|^{2}$

where $k_{1}=\left(k_{\Uparrow}^{2}-\varepsilon_{1} \omega^{2} / c^{2}\right)^{1 / 2}, \alpha_{1}=\varepsilon_{1} / k_{1}$,

$\beta_{z}=c \sqrt{\varepsilon_{2}}\left(\omega \cos \theta_{i}\right)^{-1}$.

This equation is obtained in the suppositions $e^{-2 k_{1} d}<<1$ and $\left|\varepsilon^{\prime}\right| / \varepsilon^{\prime \prime}>>1,\left|\varepsilon^{\prime}\right|>>1$ for $\omega=\omega_{s}\left(k_{\Uparrow}\right)$. Under the first condition, the law of dispersion of surface electromagnetic waves determines the spectrum of surface electromagnetic waves $\omega_{s}\left(k_{\Uparrow}\right)$.

The first condition secures small radiation damping of surface electromagnetic waves, and under the experimental conditions (when $k_{1} d<<1$ ) it is not too strong or strict. Equation (15) represents the fact that, upon the excitation of surface electromagnetic waves on smooth surface, there exists a resonant enhancement of the electric field, and its magnitude is characterized by the factor $\left|\varepsilon^{\prime}\right| / \varepsilon^{\prime \prime}$, similarly to the situation for local plasmon vibrations. The possibility of the electric field enhancement by polaritons at the flat surface and its manifestations in optical surface were considered theoretically for such processes as absorption of light by molecules near resonance with surface plasmons and RS by molecules in the ATR geometry. The consideration of the mechanism of the electric field enhancement by surface polaritons predicts an essentially smaller enhancement than the electric field enhancement due to local plasmon oscillations. For this reason, there exists an interest in attempts to separate the contributions from local plasmon oscillations and surface electromagnetic waves into the enhancement of the RS by molecules adsorbed on the rough surface [1].

The theoretical study of the enhancement effect revealed that the magnitude of the electric field enhancement $g\left(\omega_{r}\right)$ (which is $g\left(\omega_{r}\right) \sim \omega_{p} / \gamma \sim\left|\varepsilon^{\prime}\right| / \varepsilon^{\prime \prime}$ at resonance) is characterized by the concurrence of the processes of excitation and decay of surface electromagnetic waves or modes (SEM). SEM are the Bose excitation that transforms to classical modes for great numbers of occupation. For this reason, the level of pumping these SEM (in the specified external field $E_{0}$ ) is limited only by the processes of their decay. As shown in [16], when describing SEM using the dielectric function, the parameter $1 / \tau$ describes the full rate of the SEM decay: 


$$
1 / \tau=1 / \tau_{d}+1 / \tau_{s}+1 / \tau_{r}
$$

Here we single out the following channels of the SEM decay:

1) dissipative (relaxation time $\tau_{d}$ ), connected with transformation of the SEM energy into heat;

2) radiative (decay to photons for time $\tau_{r}$ );

3) surface (scattering of SEM to other SEM for time $\tau_{s}$ ).

In this connection, one should note that the observed efficiency of the electric field enhancement by local plasmon oscillations and surface electromagnetic waves could be connected with different influence of the roughness on local and delocalized plasmon oscillations. Indeed, when creating the roughness of surface with the scale smaller than the light wavelength, there increases the possibility of formation of local plasmon vibrations. However, this is accompanied by rapid decrease of the lifetime of surface electromagnetic waves and enhancement of electric field due to them, which is determined by the processes of their radiation decay and scattering by the roughness [5].

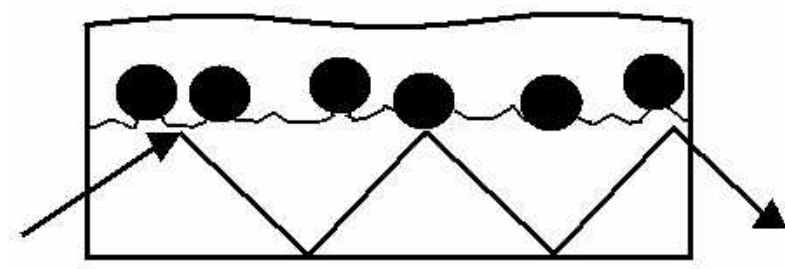

Fig. 15. Scheme of the RATR method.

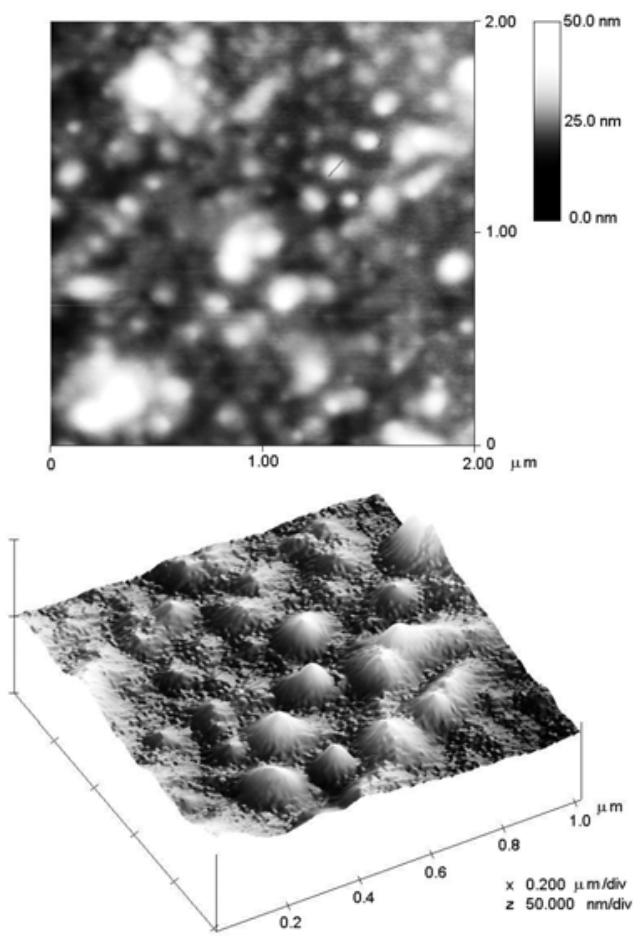

Fig. 16. 2D (a) and 3D (b) AFM images of colloidal gold on $\mathrm{Au} / \mathrm{SiO}_{2}$ substrate.

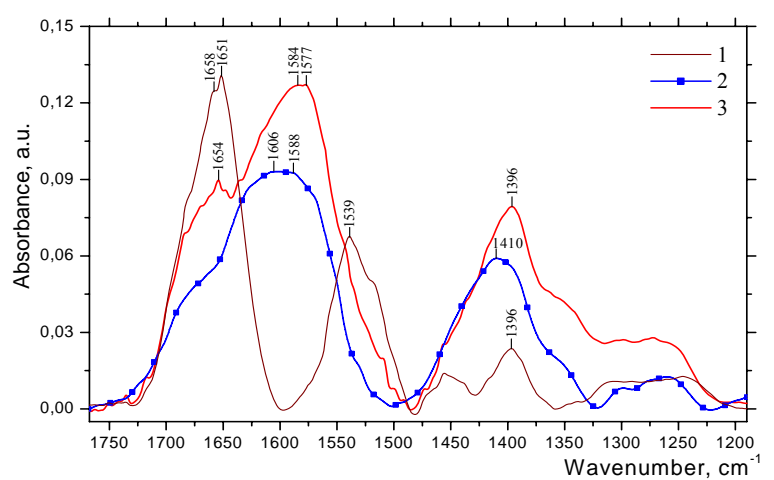

Fig. 17. SEIRA spectra of BSA (1), colloidal gold (2) and BSA - colloidal gold system (3) on the gold substrate.

\subsection{Effect of enhancement on colloid particles of gold or silver}

The third method realizes the enhancement effect on gold or silver colloid particles.

For our purpose, one can use the geometry of transmission or one can increase the sensitivity and use the repeated attenuated total reflection (Fig. 15). Or one can create a colloidal solution where the substance under study is being adsorbed on the colloidal particles. We observed the effect of enhancement of the absorbance intensity of different molecular groups near the colloidal particles.

Colloidal gold (it was produced by reduction of $\mathrm{Au}$ from $\mathrm{HAuCl}_{4}$ with citrate, concentration $12.1 \mathrm{~g} / \mathrm{l}$ ) was mixed with the aqueous solution of BSA (Sigma) $1 \ldots 40 \mathrm{~g} / 1$, then precipitated at the gold substrate and liophilically dried.

Colloidal gold is often used as an optical enhancer for IR and SPR. However, in the case of IR, the addition of colloidal gold to protein solution could complicate the FTIR spectra analysis (Fig. 16). Intensive absorption of colloidal gold about $1600 \mathrm{~cm}^{-1}$ (vibration of $\mathrm{COO}^{-}$molecular groups) (Fig. 17, curve 2) overlaps the amid I at $1651 \mathrm{~cm}^{-1}$ and amid II bands at $1539 \mathrm{~cm}^{-1}$ (Fig. 17, curve 1), that lead to difficulties in the spectra interpretation.

Maximum of amid I band in SEIRA spectra of BSA - colloidal gold system (Fig. 17, curve 3) is located at $1654 \mathrm{~cm}^{-1}$ in comparison with BSA $1651 \mathrm{~cm}^{-1}$. So, the $3 \mathrm{~cm}^{-1}$ shift is observed in SEIRA spectra of BSA - colloidal gold system and other changes in structure could be studied. So, concentration of colloidal gold would be decreased for SEIRA studies of such systems, and sometimes it could be not enough for the enhancement. That is why the colloidal gold is not a suitable enhancer for characterization of amid I and amid II bands.

In [21], the authors studied the proteins, which were adsorbed at colloidal particles (golden ones, with the average diameter of $15 \ldots 30 \mathrm{~nm}$ ). The authors obtained the dependence of the enhancement 
coefficient on the size and the type of the particles. The results showed that the SEIRA method is sensitive to interaction of protein molecules adsorbed on colloid particles with specific proteins, which make it possible to easily monitor the antigen-antibody type interactions. The obtained results can give a basis for revealing various biospecific interactions in the systems under study.

For theoretical description of this effect, we use the model of isolated particles [1]. Consider the electric field enhancement by a metal sphere with the radius $r=a$ and frequency-dependent complex dielectric permeability $\varepsilon(\omega)$; the sphere is placed in the medium with the dielectric permeability $\varepsilon_{1}$.

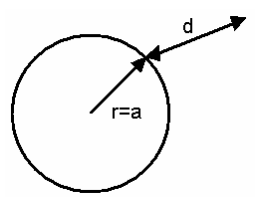

For this model, the field of dipole vibrations of electric type asymptotically transforms to flat wave with amplitude $E_{0}(\omega)$; in quasistatic approximation, it has the form:

$E(r, t)=\exp (-i \omega t)\left[E_{0}(\omega)+E_{1}(r, t)\right]$

where $E_{1}(r, \omega)=\frac{\varepsilon-\varepsilon_{1}}{\varepsilon+2 \varepsilon_{1}}\left(\frac{\alpha}{r}\right) \frac{3\left(E_{0} r\right) r-E_{0} r^{2}}{r^{2}}$,

when $r \geq \alpha, r$ is calculated starting from the center of the sphere.

The efficiency of the electric field enhancement by the sphere is characterized by the maximal value of the coefficient, which can be calculated using Eq. (10).

Table 3. Dependence of the enhancement factor on the wavenumber for membranes taken from [22] and our calculation data.

\begin{tabular}{|c|c|c|c|c|}
\hline \multirow{2}{*}{$\begin{array}{c}\text { Wavenumber, } \\
\mathrm{cm}^{-1}\end{array}$} & \multicolumn{3}{|c|}{ Enhancement factor } & \multirow{2}{*}{ Assignment } \\
\cline { 2 - 4 } & experiment & \multicolumn{2}{|c|}{ calculation } & \\
\cline { 2 - 4 } & $g^{2}$ & $G$ & $g^{2}$ & str. C=O \\
\hline 1732 & 14.9 & 3.9 & 15.2 & amid I \\
\hline 1647 & 1.4 & 3.7 & 13.7 & amid II \\
\hline 1534 & 1.6 & 3.5 & 12.3 & def. $\left(\mathrm{CH}_{3}\right)$ asym. \\
\hline 1455 & 3.1 & 3.4 & 11.6 & background \\
\hline 1384 & - & 3.2 & 10.2 & lipid (str. PO $\left.{ }_{2}^{-}\right)$ \\
\hline 1225 & 2.8 & 2.9 & 8.4 & str. (C-C ) \\
\hline 1053 & 18.5 & 2.6 & 6.8 & \\
\hline
\end{tabular}

Table 4. Characteristics of the enhancement factor of electric field formula (10) at excitation of surface electromagnetic modes taken from [5].

\begin{tabular}{|c|c|c|l|l|}
\hline & \multicolumn{3}{|c|}{ Local plasmon vibrations } & $\begin{array}{c}\text { Surface electromagnetic } \\
\text { modes }\end{array}$ \\
\hline $\begin{array}{c}\text { Decrease of } g \text { with distance } \\
\text { under moving off the } \\
\text { surface }\end{array}$ & $\sim(a / r)^{3}$ & $\sim(a / r)^{3}$ & $\begin{array}{l}\text { At overmicroscopical scale } \\
\sim 10^{4} \AA[26]\end{array}$ & $\begin{array}{l}\text { Excitation theory: } \\
\sim \exp \left(-|z| \sqrt{q^{2} \|-\omega^{2} / c^{2}}\right), \\
\text { numerical computation:[28] }\end{array}$ \\
\hline
\end{tabular}

Table 5. Advantages and disadvantages of the SEIRA method.

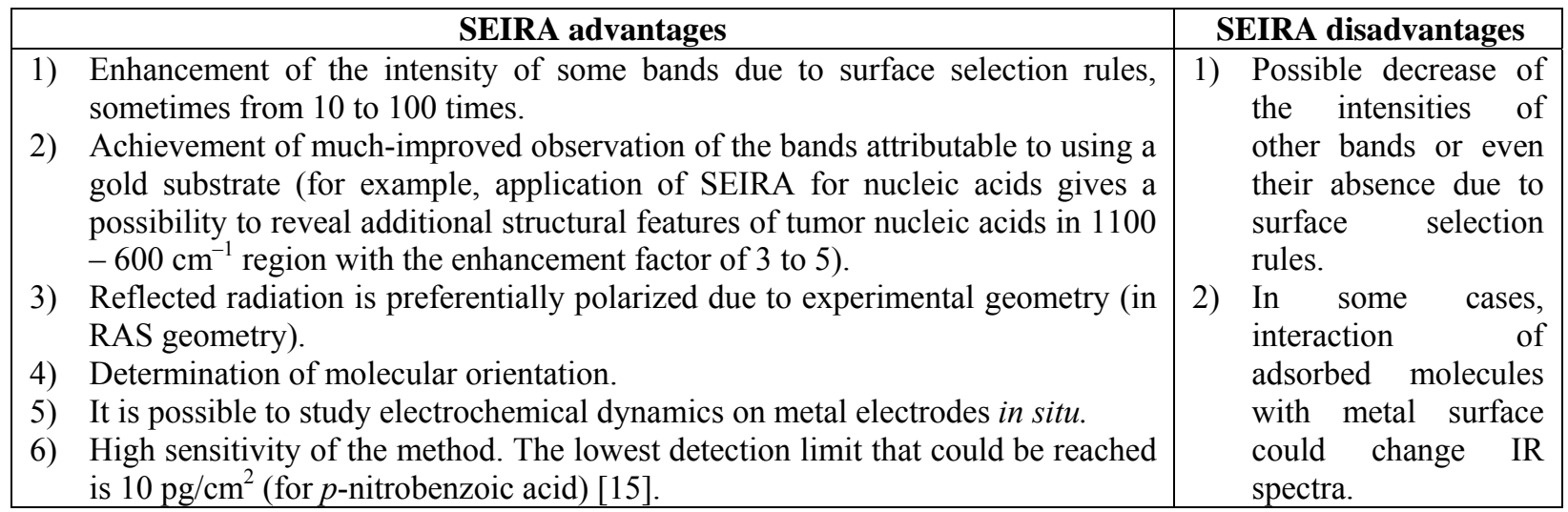




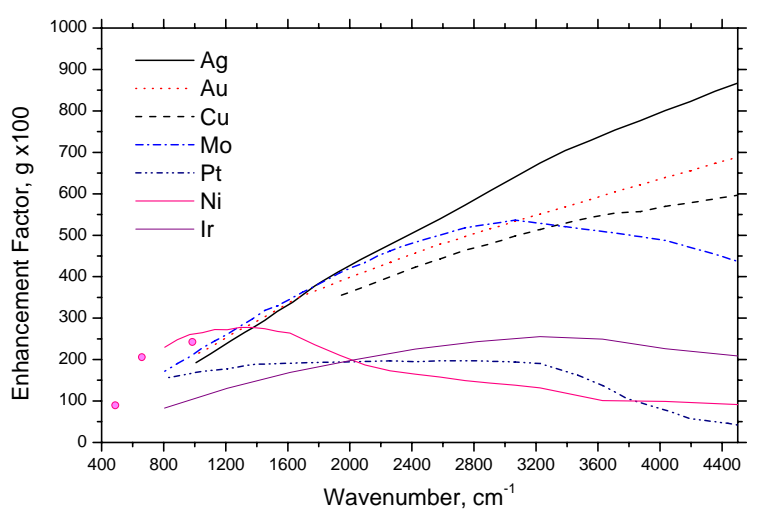

Fig. 18. Enhancement factor for various metals and graphite (indicated by points).

In the pole of the sphere $\left(r=a e_{0}\right.$, where $\left.e_{0}=E / E_{0}\right)$, we have:

$g^{2}(\omega)=\left|\frac{E\left(a e_{0}, \omega\right)}{E_{0}(\omega)}\right|=\frac{9|\varepsilon(\omega)|^{2}}{\left[\varepsilon^{\prime}(\omega)+2 \varepsilon_{1}\right]^{2}+\left[\varepsilon^{\prime \prime}(\omega)\right]^{2}}$.

Here $\varepsilon^{\prime}=\operatorname{Re}(\varepsilon), \quad \varepsilon^{\prime \prime}=\operatorname{Im}(\varepsilon)$ and $g(\omega)$ is the enhancement coefficient.

In Table 3, we present the enhancement factor from the experiment [22] in RATR (repeated attenuated total reflection) geometry for biological membranes into which the colloid particles of silver are introduced (Fig. 15).

\section{Factor of enhancement in SEIRA experiment}

Earlier in our experiments [17, 18] and in literature [22] we found that the SEIRA factor was equal to $3 \ldots 20$ for vibrations of different molecular groups in DNA and membranes (proteins and lipids). As usual, the calculated factor $g \sim \varepsilon^{\prime} / \varepsilon^{\prime \prime}$ does not coincide with that experimentally obtained. The possible reason is the fact that we do not take into account the change in the polarizability of the molecules absorbed on the metal surface.

From analysis of the formulae (15) the factor of enhancement $g^{2}$ is proportional to $\sim\left(\varepsilon^{\prime} / \varepsilon^{\prime \prime}\right)^{2}$. We estimated $g$ for different metals and could conclude that silver, gold and copper are the best from the enhancers for optical transition in a wide IR region (Fig. 18). Once our experimental data [23] put a question: could graphite and/or carbon nanotube show a property of IR enhancer? Estimation showed that in IR region $(10 \ldots 20 \mu \mathrm{m})$ their dielectric properties could give the enhancement factor $g^{2}$ within the range of 2 to 6 [24]. These data are presented in Fig. 19 by separated points. In our experiment, we registered the enhancement factor which was equal 1.4 to 9 for vibrations of residual graphite on the surface of carbon nanotubes in IR experiments.

Many factors influence this effect (geometry of the experiment, type of bond between surface and adsorbed molecules, dielectric properties of the molecules and

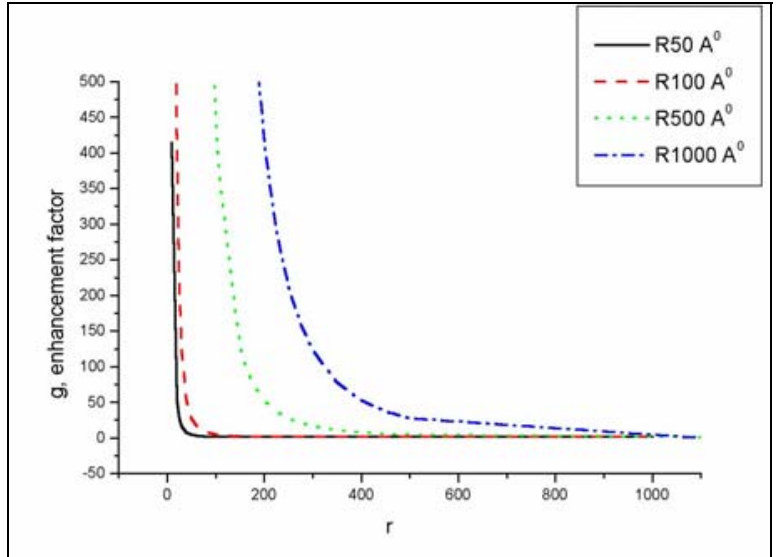

Fig. 19. Dependence on the enhancement factor $g$ of the local field on the distance $r$ from the surface with gold spheres of various sizes $(50,100,500,1000 \AA)$.

environment, type of metal surface etc.). We will try to reply to other question - about the decay of local field enhancement induced by metal surface. Longrange and short-range interaction mechanisms of the local field enhancement could influence the process of the optical signal enhancement.

Short-range enhancement. If we apply to [6], the authors write that in the case when the local plasmon field is responsible for giant Raman scattering (GRS), a small moving away the molecule from the metal surface does not drastically influence the GRS (in contrast of the chemical bond of the molecule with metal surface). Under the moving away the molecules not far (distance $R$ ) from the surface, the enhancement factor $G$ (that is proportional to $E^{4}$ for $\mathrm{RS}$ and $E^{2}$ for IR) is decreased according to the formula $G \sim\left(r_{0} / R\right)^{12}$, where $r_{0}$ is the characteristic geometrical size of the roughness. For example, for the polarized sphere of the radius $r_{0}$ the Coulomb field $E$ is equal to $E \sim r_{0}^{3} / R^{3}$, consequently, the $d_{s} \sim\left(r_{0} / R\right)^{3}$. Due to the fact that $d_{s} \sim \widehat{r} E_{l o c}$; $E_{\text {loc }}=L\left(\omega_{i}\right) E_{i} . D_{\text {eff }}\left(\omega_{s}\right) \sim\left(r_{0} / R\right)^{6}$ and $G \sim\left(r_{0} / R\right)^{12}$. It means that the GRS effect could be registered for several monolayaers of the molecules adsorbed at the metal surface.

The dependence of the GRS on the distance of the molecules adsorbed at the metal surfaces is studied in the [6]. It was shown that removal of the molecules from the surface to $50 \ldots 100 \AA$ does not essentially change the intensity of the GRS.

The dependence of the enhancement factor on the distance for long-interaction mechanism of enhancement was estimated in [25] where detected was the change in the RS intensity under increasing the number of the molecular layers adsorbed on the surface. The results correspond to the forecast that states that the intensity of GRS should decrease exponentially with increasing of the distance from the surface. The characteristic length of this decay is 
equal to the characteristic decrease of the surface plasmon field.

In [5] these data are presented in Table 4, one of its lines is presented below.

We estimated the decay of the local field (in the medium with $\varepsilon=1$ and at the distance $r$ ) induced by external electromagnetic field in the surface gold sphere of varies sizes. The results are presented in Fig. 19.

$g=\left|\frac{E}{E_{0}}\right|=1+3.3\left(\frac{\alpha}{r}\right)^{3} \sqrt{\frac{\varepsilon^{\prime}-2 \varepsilon^{\prime}+1+2 \varepsilon^{\prime \prime}}{\varepsilon^{\prime 2}-4 \varepsilon^{\prime}+4+\varepsilon^{\prime \prime 2}}}$, where $a-$ radius of metal sphere that is assigned by $R$ inside Fig. 1, $r$ - distance from the metal sphere, $\varepsilon^{\prime}$ - real and $\varepsilon^{\prime \prime}-$ imaginary parts of the dielectric permeability of metal spheres.

So, this estimation should be also true for SEIRA and shows that the distance could be $100 \ldots 1000 \AA$ and even more. It depends on many experimental factors.

In Table 5, we present advantages and disadvantages SEIRA method.

\section{Conclusions}

To summarize, different methods of enhancement for optical transitions of molecules near rough or curve metal surface such as SERS, SEIRA and metal enhanced luminescence are presented here. The mechanism of enhancement is based on the enhancement of electric field near metal surface with curvature and as a result, an increase of the section of the opical processes, as well as an increase of dipol moments of molecular groups of the molucules at metal surface. In many cases these methods are non-competitive, they are complementary and could be effictively used for probing of extra small amounts of substances, including submonolayers and separate molecules. SEIRA and metal enhanced luminescence are preferred for macromolecules, as well as SERS is more proper for study of small molecules and its interactions.

References

1. V.A. Kosobukin // Surface. Physics, chemistry, mechanics 12, p. 5-20 (1983).

2. V.V. Bondar, M.V. Kuryk // J. Exp. Theor. Phys. 78 (1), p. 94-99 (1980).

3. M. Fleischman, P.J. Hendra and A.J. McQuillan // Chem. Phys. Lett. 26, p.123 (1974).

4. K. Kneipp, H. Kneipp, I. Itzkan, R.R Dasari and M.S Feld // J. Phys.: Condens. Matter 14, p. 597-624 (2002).

5. V.A. Kosobukin // Reports of Academy of Sciences of USSR, Physical edition 48 (7), p.1281-1288 (1984).

6. V.I. Emelyanov, N.I. Koroteev // Uspekhi Fizicheskikh Nauk 135 (2), p. 345-361 (1981).

7. D.A. Zwemer, J.E. Rowe, C.V. Shank, J.R. Murray // Proc. VII Intern. Conf. on Raman Spectroscopy, ed. W.H. Murphy, Amsterdam, N.Y.; North-Holland, p. 414 (1980).
8. D.Ch. Geddes, J.R. Lakowicz // J. Fluoresc.12 (2), p.121-129 (2002).

9. A. Camplon, A.R. Gallo, C.B. Harris, H.J. Robota, and P.M. Whitmore // Chem. Phys. Lett. 73 (3), p. 447-450 (1980).

10. J. Kummerlen, A. Leitner, H. Brunner, F.R. Aussenegg, and A. Wokaun // Mol. Phys. 80 (5), p. 1031-1046 (1993).

11. K. Sokolov, G. Chumanov and T.M. Cotton // Anal. Chem. 70, p. 3898-3905 (1998).

12. T. Hayakawa, S.T. Selvan and M. Nogami // Appl. Phys. Lett. 74 (11), p. 1513-1515 (1999).

13. J.R. Lakowicz // Anal. Biochem. 298, p.1-24 (2001).

14. I. Gryczynski, J. Malika, Z. Gryczynski, C.D. Geddes and J.R. Lakowicz // J. Fluoresc. 12 (1), p. 11-13 (2002).

15. M. Osawa, Surface-enhanced infrared absorption spectroscopy, Handbook of vibrational spectroscopy, eds J.M. Chalmers and P.R. Griffiths, vol. 1, p. 85-799 (2002).

16. V.A. Kosobukin // Reports of Academy of Sciences of USSR, Physical edition, 49 (6), p. 1111-1120 (1985).

17. G.I. Dovbeshko, V.I. Chegel, N.Y. Gridina, O.P. Repnytska, Y.M. Shirshov, V.P. Tryndiak, I.M. Todor, G.I. Solyanik // Biospectroscopy, p. 470486 (2002).

18. G.I. Dovbeshko, V.I. Chegel, N.Y. Gridina, O.P. Repnytska, I.V. Sekirin, Y.M. Shirshov // Proc. SPIE, eds S. Sveshnikov, S. Kostyukevich 4425, p. 158-162 (2001).

19. E.D. Palik (ed.) Handbook of optical constants of solids, p. 804 (1985).

20. A. Pelmenschikov, D. Hovorun, O. Shishkin, J. Leszczynski // J. Chem. Phys. 113 (14), p. 59865990 (2000).

21. A.A. Kamnev, L.A. Dykman, P.A Tarantilis, M.G. Polissio // Eucmos XXVI - Villeneuve D’ASCO, p. 23 (2002).

22. Ch. Kuhne, G. Steiner, W.B. Fischer and R. Salzer // Fresenius J. Anal. Chem. 360, p. 750754 (1998).

23. G.I. Dovbeshko, O.P. Repnytska, E.D. Obraztsova, Y.V. Shtogun // Chem. Phys. Lett. 372, p. $432-437$ (2003).

24. V.P. Tomaselli, R. Rivera, D.C. Edewaard, K.D. Molier // Appl. Opt. 20 (22), p. 3961-3967 (1981).

25. P.N Sanda, J.M. Warlaumont, J.E Demuth, J.C Tsang, K Christman, J.A Bradley // Phys. Rev. Lett. 45, p. 1519 (1980).

26. R. Ruppin // Solid State Communs 39, p. 903 (1981).

27. P.S. Das, J.I. Gersten // Phys. Rev. B25, p. 6281 (1982).

28. S.S. Jha, J.R. Kirtley, J.C. Tsang // Phys. Rev. B22, p. 3973 (1980). 
Semiconductor Physics, Quantum Electronics \& Optoelectronics. 2004. V. 7, N 4. P. 411-424.

(C) 2004, V. Lashkaryov Institute of Semiconductor Physics, National Academy of Sciences of Ukraine 